\title{
Broadband and Picosecond Intraband Absorption in Lead based Colloidal Quantum Dots
}

\author{
Bram De Geyter ${ }^{1,2}$, Pieter Geiregat ${ }^{1,2}$, Yunan Gao ${ }^{3,4}$, Sybren ten Cate ${ }^{3}$, Arjan J. Houtepen ${ }^{3}$, Juleon M. \\ Schins $^{3}$, Dries Van Thourhout ${ }^{1}$, Laurens D.A. Siebbeles ${ }^{3}$, and Zeger Hens ${ }^{5}$ \\ ${ }^{1}$ Photonics Research Group, INTEC Department, Ghent University-IMEC, Sint-Pietersnieuwstraat 41, 9000 \\ Ghent, Belgium \\ ${ }^{2}$ Center for Nano- and Biophotonics (NB-Photonics), Ghent University, 9000 Ghent, Belgium \\ ${ }^{3}$ Optoeletronic Materials Section, Department of Chemical Engineering, Delft University of Technology, \\ Julianalaan 136, 2628 BL Delft, The Netherlands \\ ${ }^{4}$ The Kavli Institute of Nanoscience, Delft University of Technology, Lorentzweg 1, 2628 CJ Delft, The \\ Netherlands \\ ${ }^{5}$ Inorganic and Physical Chemistry Departement, UGent, Krijgslaan 281 (S3), B-9000 Gent, Belgium
}

\begin{abstract}
Using femtosecond transient absorption spectroscopy we demonstrate that lead chalcogenide nanocrystals show efficient, photoinduced absorption (PA) in a broad wavelength range starting just below the bandgap. The timedependent decay of the PA signal correlates well with the recovery of the band gap absorption. Therefore, the same carriers are involved, which decay with the typical Auger recombination rate in these nanocrystals. Based on this, we assign this PA signal to intraband absorption, i.e., the excitation of photogenerated carriers from the bottom of the conduction band or the top of the valence band to higher energy levels in the conduction and valence band continuum. This broadband response in the commercially interesting near to mid-infrared range is very relevant for ultra high speed all optical signal processing.

Keywords:intraband absorption, colloidal nanocrystals, transient absorption spectroscopy, lead chalcogenide, tight-binding, free carrier absorption, optical signal processing
\end{abstract}

\section{INTRODUCTION}

The tunability and ease of processing of colloidal quantum dots (CQDs) offer many opportunities for the development of novel opto-electronic devices, such as lasers, electro-optic modulators and high-speed detectors[1, 2]. The design of efficient and competitive materials for the photonics industry based on CQDs requires a precise understanding of the electronic states involved in light-matter interaction and the decay dynamics of photogenerated carriers. In the case of CQDs with their first exciton absorption in the near infrared (800-2000 nm), most studies have focused in this respect on interband transitions and intraband relaxation[3, 4, 5, 6]. However, a number of literature studies indicate that additional electronic transitions are relevant in excited CQDs.

\subsection{Rationale}

Intraband absorption between $1 \mathrm{~S}$ and $1 \mathrm{P}$ state has been studied extensively for both $\mathrm{CdSe}[7,8,9], \mathrm{PbSe}[6,10]$ and $\mathrm{PbS}$ [11]. This allowed transition is therefore well understood, with excellent agreement between theory and experiment. In the case of CdSe QDs, Malko et al.[12] reported photoinduced absorption (PA) at wavelengths directly below the band gap, which they attributed to absorption from excited trap states at the CQD surface.

\subsection{Outline}

In this paper we report on strong, photo-induced absorption (PA) in lead chalcogenide CQDs in a broad wavelength range between 1400 and $2000 \mathrm{~nm}(0.5 \mathrm{eV}$ to $0.85 \mathrm{eV})$ starting directly below the bandgap. Using femtosecond transient absorption (TA) spectroscopy, we find that the decay of the PA matches the recovery of the band gap absorption. Therefore, we interpret the PA in terms of transitions of excited electrons and holes from the lowest conduction (LUMO) and the highest valence band state (HOMO) to the conduction and valence band continuum, respectively. This broad intraband absorption is a characteristic of several $\mathrm{Pb}$ chalcogenide CQDs, such as $\mathrm{PbS}$, $\mathrm{PbSe}$ and $\mathrm{PbSe} / \mathrm{CdSe}$. Moreover, extending over the wavelength range 1400 and $2000 \mathrm{~nm}(0.5$ to $0.89 \mathrm{eV})$, the observation of photoinduced absorption in lead chalcogenide CQDs is highly relevant to the design of CQD-based materials for telecom applications, which operate in this wavelength range. Finally, in bulk semiconductors high energy intraband transitions are forbidden, because of momentum conservation. In the case of a particle in a infinite 
(a)
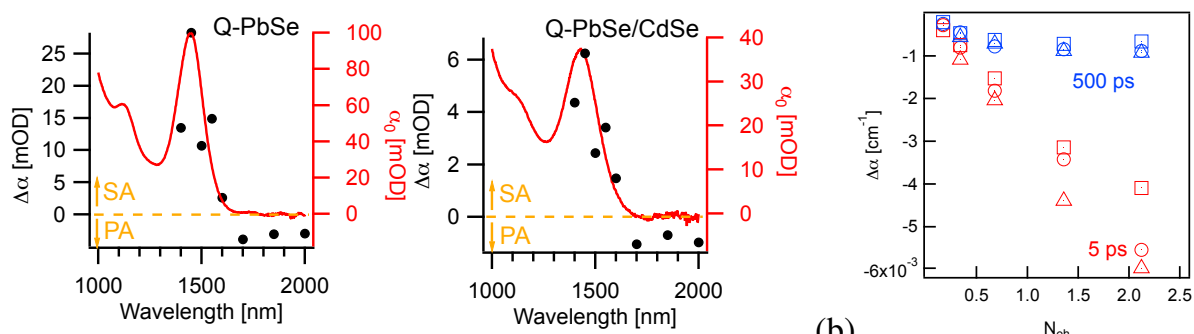

(b)

$\mathrm{N}_{\mathrm{eh}}$

(c)

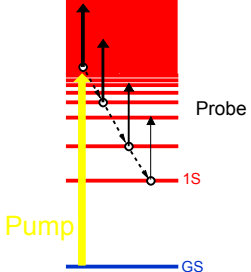

Figure 1: (a)Steady state (red line, right axis) and differential transient absorption spectrum (black dots, left axis) of $\mathrm{PbSe} \mathrm{CQDs}$ (left graph) and $\mathrm{PbSe} / \mathrm{CdSe}$ (right graph) 5 ps after the pump pulse shows absorption bleach (AB) of the band gap absorption $\left(\Delta \alpha=\alpha_{0}-\alpha>0\right)$ and wavelength-independent photoinduced absorption (PA, $\left.\Delta \alpha<0\right)$ below the band gap.(b) Differential absorption as a function of average number of excitons $N_{e h} 5 \mathrm{ps}$ after the pump pulse (red) and $500 \mathrm{ps}$ after the pump pulse (blue) at $1700 \mathrm{~nm}$ (triangles), $1850 \mathrm{~nm}$ (squares) and $2000 \mathrm{~nm}$ (circles) for PbSe QDs. (c) Graphical representation of the fast dynamics observed in the PA probe signal after the pump pulse.

spherical potential well, only transitions with orbital angular momentum $\Delta l= \pm 1$ are allowed. By proving that the observed PA corresponds to intraband absorption, comparable in strength to the bandgap transition, our results are a direct manifestation of quantum confinement effects on semiconductor materials and therefore appealing from a theoretical viewpoint.

\section{RESULTS}

Figure 1 shows the change in absorption of PbSe core $(d=4.6 \mathrm{~nm})$ and $\mathrm{PbSe} / \mathrm{CdSe}$ core/shell $(4.5 \mathrm{~nm}$ core, $0.5 \mathrm{~nm}$ shell $)$ following excitation with a $50 \mathrm{fs}$ laser pulse $\left(\lambda_{\text {pulse }}=790 \mathrm{~nm}\right)$. This resulting excited state cools down rapidly, where the electron and the hole relax to the LUMO and HOMO, respectively[13]. A buildup of carriers at the band gap reduces the band gap absorption (absorption bleach, AB), as can be seen in Fig.1. Here, a bleach is indicated by a positive value for $\Delta \alpha=\alpha_{0}-\alpha$, where $\alpha_{0}$ is the steady state absorbance and $\alpha$ is the absorbance after the pump pulse. However, below the bandgap, from $1700 \mathrm{~nm}$ up to $2000 \mathrm{~nm}$, a negative signal, indicating photoinduced absorption (PA) for both materials.

To gain insight into the nature and strength of the PA, we performed both power and wavelength dependent measurements. At high fluences, when more than one exciton is created per QD, the initial exciton relaxation is governed by Auger recombination[14]. After 500 ps all multiexcitons have recombined, leaving a population of singly excited QDs. The normalized differential absorption after 500 ps therefore becomes, assuming a Poissonian distribution of carriers:

$$
\frac{\Delta \alpha_{500 p s}}{\alpha_{0}}=\frac{2}{g}\left(1-e^{-N_{e h}}\right)
$$

Here we have expressed the pump power as the average number $N_{e h}$ of excitons that are created per QD by the pump pulse. $g$ is the degeneracy of the LUMO and the HOMO. The factor two accounts for both electrons and holes, since the differential absorption is proportional to the sum of all involved carriers.

The power dependence of the PA for short and long times after the pump pulse is given in Fig. 1.a for PbSe. A similar behaviour is observed for $\mathrm{PbSe} / \mathrm{CdSe}$. At short delay times (red curves), the PA follows a linear trend within the given power range. At long times, we observe a Poissonian trend, which fits well to Eq. 1.

Based on these traces, we quantify the strength of the PA transition as the absorbance per exciton $\alpha_{P A, e x}$. This is the maximum PA after long delay, obtained from fitting Eq. 1 to the blue curves in Fig. 1.a (see Table. 1). Compared to the band gap bleach per exciton for $\mathrm{PbSe} \alpha_{A B, e x}\left(125 \cdot 10^{-4} \mathrm{~cm}^{-1}\right)$, this transition is about 20 times less strong. The PA per exciton $\alpha_{P A \text {,ex }}$ in $\mathrm{PbSe} / \mathrm{CdSe}$ is about 10 times weaker than the $\alpha_{A B \text {,ex }}\left(44 \cdot 10^{-4} \mathrm{~cm}^{-1}\right)$. To compare both materials, the absorption coefficient $\mu[15]$ for the PA is also given in Table 1. It is lower for $\mathrm{PbSe} / \mathrm{CdSe} \mathrm{QDs}$ than for PbSe QDs. Given the increasing local field factor in core/shell CQDs compared to core only QDs[16], this translates in a lower oscillator strength for PbSe/CdSe QDs.

Figure 2 shows that the transient absorption for PbSe at the band gap maximum (red trace) and below the band gap (black traces, inverted for clarity) follow a very similar time dependence after 10 ps delay for three different values of the pump power. Fitting a sum of three exponentials to the absorption transients (see Supporting Information about the fitting procedure) yields a biexciton lifetime of $82 \mathrm{ps}$, a triexciton lifetime of $32 \mathrm{ps}$ and a quadrexciton lifetime of 13 ps for the PbSe CQDs at the bandgap. The PA transients however show different dynamics at early delay times. The onset is much steeper, especially for the highest pump power, and even for low 


\begin{tabular}{|c|c|c|c|c|}
\hline & \multicolumn{2}{|c|}{$\mathrm{PbSe}$} & \multicolumn{2}{c|}{$\mathrm{PbSe} / \mathrm{CdSe}$} \\
\hline$\lambda_{\text {probe }}$ & $\alpha_{P A, e x}$ & $\mu_{P A, e x}$ & $\alpha_{P A, e x}$ & $\mu_{P A, e x}$ \\
\hline$[\mathrm{nm}]$ & {$\left[\mathrm{cm}^{-1}\right]$} & {$\left[\mathrm{cm}^{-1}\right]$} & {$\left[\mathrm{cm}^{-1}\right]$} & {$\left[\mathrm{cm}^{-1}\right]$} \\
\hline \hline 1700 & $7.1 \cdot 10^{-4}$ & 83 & $4.3 \cdot 10^{-4}$ & 47 \\
\hline 1850 & $5.6 \cdot 10^{-4}$ & 66 & $3.8 \cdot 10^{-4}$ & 41 \\
\hline 2000 & $7.5 \cdot 10^{-4}$ & 88 & $4.3 \cdot 10^{-4}$ & 47 \\
\hline
\end{tabular}

Table 1: Photoinduced absorbance for a population of one exciton in the initial state $\alpha_{P A \text {,ex }}$, derived from a Poissonian fit, together with the absorption coefficient for $\mathrm{PbSe}$ and $\mathrm{PbSe} / \mathrm{CdSe} \mathrm{QDs}$.

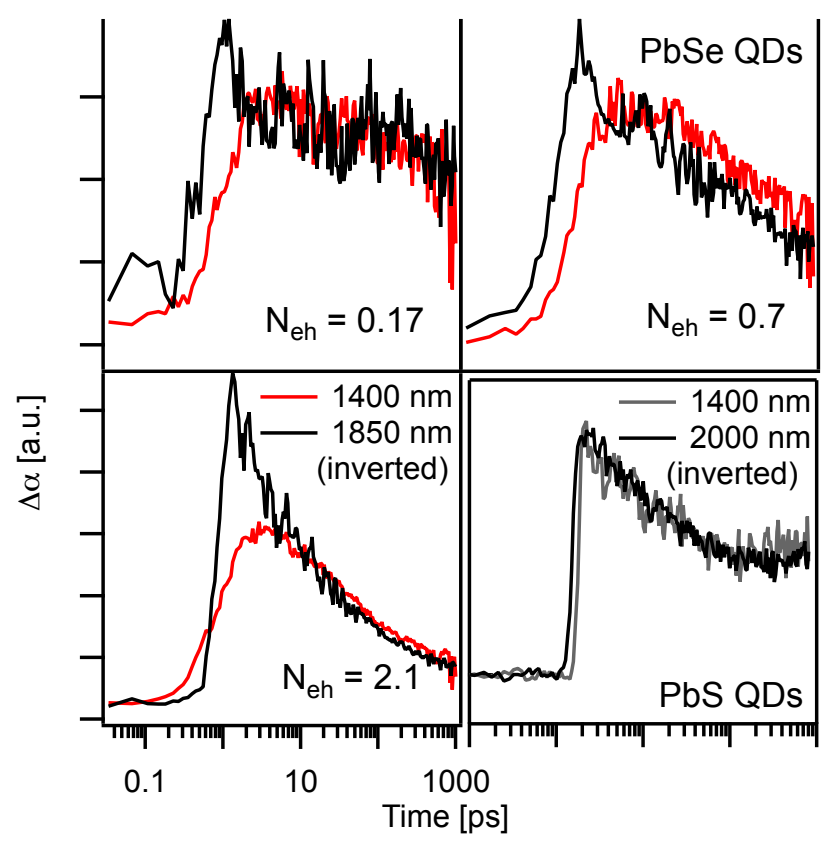

Figure 2: (a) Absorption transients for the differential absorption at the band gap (1450 nm, red) and below the band gap (1850 nm, black, inverted for clarity) for PbSe QDs show similar decay dynamics after 10 ps for three different values of the pump power. An initial faster decay is present in the PA transients for all three pump powers shown. (b) Absorption transients for very small PbS CQDs $(2.45 \mathrm{~nm})$ for which the pump is resonant with the band gap transition $(780 \mathrm{~nm})$ show PA $(\Delta \alpha<0)$, yet no fast initial decay component.

pump powers, an initial decay is present, which is not observed in the differential absorption transients around the bandgap. The fitting procedure yields a lifetime of $1.2 \mathrm{ps}$ for this initial decay. For PbSe/CdSe QDs, which show a similar dynamics, we extract a biexciton lifetime of 84 ps and a triexciton lifetime of 16 ps. For the PA transients we extract an initial decay time of 1.3 ps.

\section{DISCUSSION}

The wavelength-independent PA we observe in these near-infrared CQDs can have different origins, such as (1) Coulomb shifts in the multiexcitonic absorption spectrum, (2) absorption from surface-localized states or defects, or (3) intraband absorption. Coulomb shifts can be excluded, since the PA is observed up to $2000 \mathrm{~nm}$, well below the band gap. Moreover, a spectral dependency similar to the band gap absorption would be observed in the case of Coulomb shifts. Malko et al.[12]reported on PA in transient absorption transients on colloidal CdSe QDs.

An extensive study with different sizes and surface compositions revealed a strong dependence on solvent and surface passivation, along with a low size dependence of the PA cross section. Given the high PA cross section, they therefore attribute it to transitions from dangling surface bonds to excited states in the capping or surrounding matrix, rather than intraband absorption. Here, we work with a considerably lower photon energy $(0.5$ to $0.8 \mathrm{eV}$ vs. 2.0 to $2.5 \mathrm{eV}$ ), making a transition to a final state in the surrounding matrix improbable. Moreover, the PA we observe at long delay times saturates with increasing pump power, similar to the bleach of the band gap absorption. This shows that the initial state of the PA is the band gap exciton. That QD core states are involved, instead of 
surface states, is further confirmed by the Auger dynamics of the PA signal. We therefore conclude that the PA observed here finds its origin in intraband absorption.

Intraband absorption is forbidden in first order for bulk semiconductors. In quantum dots, however, the conservation of crystal momentum is relaxed, resulting in a non-zero probability for intraband absorption. The excited carriers at the LUMO and HOMO are the initial state for the intraband absorption. For long delay times, we therefore expect the same pump power dependence for band gap $\mathrm{AB}$ as for the sub gap PA, since the same carriers are involved. Moreover, we expect to observe the dynamics of Auger recombination of multiexcitons, since this dictates the rate at which carriers disappear from the LUMO and the HOMO. Both effects we have shown experimentally (see Fig. 1.a). At short delay times however the decay dynamics are not completely similar. A faster picosecond component is visible in the PA transients, together with a more immediate rise of the PA signal. The pump power dependence at short delay also becomes linear in the given power range. We attribute the quick rise time to instantaneous PA from higher energy excited states (see Fig. 2.a). The fast initial decay can be attributed to carriers cooling from higher energy states to the LUMO and the HOMO (see Fig. 2.b). This is confirmed by the PA transients of small PbS CQDs (see Fig. 2.b), where the pump is resonant with the band gap and where no initial decay is observed. The time scale we measure correlates well with other reports in literature for carrier cooling $[4,17,5]$. Moreover, the higher degeneracy of the initial excited state explains the linear power dependence we observe at short delay times (see Fig. 1.a).

\section{CONCLUSIONS}

In conclusion we have shown that intraband absorption in the telecom wavelength range is universal to quantum confined lead chalcogenide QDs. The absorption is between 10 and 20 times weaker than the band gap absorption bleach. The intraband absorption is broadband and constant over a large wavelength range. This range can be easily tuned by changing the QD size. The intraband absorption has ultrafast dynamics in the one to tens of picoseconds range, governed by carrier cooling and Auger recombination. Our work is essential to understand the response of CQDs as gain media and ultrafast nonlinear media for photonic applications. Moreover, our measurements are a clear manifestation that the selection rules for the bulk crystal momentum and for the angular momentum in spherically confined structures as mentioned above are relaxed in CQDs and are therefore very interesting from a theoretical standpoint. They go beyond intersubband absorption $[6,8,9,7,11]$ and cover a new wavelength range directly below the band gap. Intraband absorption offers a nice way to study the dynamics of carrier cooling $[4,3,5]$ in a direct way, free from possible Coulomb shifts or redistribution over dark and bright band gap states, which cloud typical TA measurements at the band gap.

\section{ACKNOWLEDGEMENTS}

This work is part of the Joint Solar Programme (JSP) of the Stichting voor Fundamenteel Onderzoek der Materie FOM, which is supported financially by the Nederlandse Organisatie voor Wetenschappelijk Onderzoek (NWO). This work is co-financed by Nuon Helianthos. This work has also been financially supported by the 3TU Centre for Sustainable Energy Technologies (Federation of the Three Universities of Technology in The Netherlands). The authors acknowledge BelSPo (IAP 6.10, photonics@be), EU-FP7 (Navolchi) and Ghent University for funding. B.D.G. acknowledges financial support by the Bijzonder Onderzoeksfonds of Ghent University. A.J.H. acknowledges financial support by the Netherlands Organisation for Scientific Research (NWO) through a NWO-VENI grant. D.V.T. acknowledges the ERC for an ERC Starting Grant (ULPPIC).

\section{References}

[1] EH Sargent. Infrared quantum dots. Advanced Materials, 17:515-522, 2005.

[2] E. Lifshitz, M. Brumer, A. Kigel, A. Sashchiuk, M. Bashouti, M. Sirota, E. Galun, Z. Burshtein, A. Q. Le Quang, I. Ledoux-Rak, and J. Zyss. Stable pbse/pbs and pbse/pbsexs1-x core-shell nanocrystal quantum dots and their applications. Journal of Physical Chemistry B, 110:25356-25365, 2006.

[3] Juleon M. Schins, M. Tuan Trinh, Arjan J. Houtepen, and Laurens D. A. Siebbeles. Probing formally forbidden optical transitions in pbse nanocrystals by time- and energy-resolved transient absorption spectroscopy. Physical Review B, 80(3), July 2009.

[4] Joonhee M. An, Marco Califano, Alberto Franceschetti, and Alex Zunger. Excited-state relaxation in pbse quantum dots. Journal of Chemical Physics, 128(16), April 2008.

[5] Jeffrey M. Harbold and Frank W. Wise. Photoluminescence spectroscopy of pbse nanocrystals. Physical Review B, 76, 2007.

[6] BL Wehrenberg, CJ Wang, and P Guyot-Sionnest. Interband and intraband optical studies of pbse colloidal quantum dots. Journal of Physical Chemistry B, 106:10634-10640, 2002. 
[7] P Guyot-Sionnest and MA Hines. Intraband transitions in semiconductor nanocrystals. Applied Physics Letters, 72(6):686-688, February 1998.

[8] CJ Wang, M Shim, and P Guyot-Sionnest. Electrochromic nanocrystal quantum dots. Science, 291(5512):2390-2392, March 2001.

[9] A. Pandey and P. Guyot-Sionnest. Intraband spectroscopy and band offsets of colloidal ii-vi core/shell structures. The Journal of chemical physics, 127:104710, 2007.

[10] J. M. An, A. Franceschetti, S. V. Dudiy, and Alex Zunger. The peculiar electronic structure of pbse quantum dots. Nano Letters, 6:2728-2735, 2006.

[11] Ye Yang, William Rodríguez-Córdoba, and Tianquan Lian. Ultrafast charge separation and recombination dynamics in lead sulfide quantum dot-methylene blue complexes probed by electron and hole intraband transitions. Journal of the American Chemical Society, 133(24):9246-9249, 2011.

[12] AV Malko, AA Mikhailovsky, MA Petruska, JA Hollingsworth, H Htoon, MG Bawendi, and VI Klimov. From amplified spontaneous emission to microring lasing using nanocrystal quantum dot solids. Applied Physics Letters, 81:1303-1305, 2002.

[13] VI Klimov and DW McBranch. Femtosecond high-sensitivity, chirp-free transient absorption spectroscopy using kilohertz lasers. Optics Letters, 23:277-279, 1998.

[14] VI Klimov, AA Mikhailovsky, DW McBranch, CA Leatherdale, and MG Bawendi. Quantization of multiparticle auger rates in semiconductor quantum dots. Science, 287:1011-1013, 2000.

[15] Iwan Moreels and Zeger Hens. On the interpretation of colloidal quantum-dot absorption spectra. Small, 4(11):1866-1868, November 2008.

[16] Bram De Geyter and Zeger Hens. The absorption coefficient of pbse/cdse core/shell colloidal quantum dots. Applied Physics Letters, 97(16), October 2010.

[17] RD Schaller, JM Pietryga, SV Goupalov, MA Petruska, SA Ivanov, and VI Klimov. Breaking the phonon bottleneck in semiconductor nanocrystals via multiphonon emission induced by intrinsic nonadiabatic interactions. Physical Review Letters, 95(19):196401, November 2005. 